\title{
Discapacidad Intelectual en Primera Infancia: La Perspectiva de los/as Hermanos/as de Niños/as con Síndrome de Down
}

\author{
Intellectual Disability in Early Childhood: The Perspective of \\ the Brothers and Sisters of Children with Down's Syndrome
}

\author{
Paola Andreucci Annunziata * \\ Camila Morales Cabello \\ Universidad Gabriela Mistral, Chile
}

\begin{abstract}
El estudio se inscribe en el ámbito de la Atención Temprana (AT). Se propuso aportar al abordaje de la discapacidad intelectual (DI), desde una perspectiva sistémicofamiliar, relevando el aporte de los agentes socio-familiares en la construcción del fenómeno. Para ello, se ocupó de otorgar voz a los/as hermanos/as de niños/as con síndrome de Down, indagando en sus representaciones socio-cognitivas (concepciones, actitudes y sentimientos) respecto de sí mismos, sus hermanos/as en situación de DI y su rol en la familia. El diseño metodológico de corte cualitativo se valió de entrevistas en profundidad orientadas por guion temático. El grupo de estudio se conformó con 18 hermanos/as chilenos/as y 2 inmigrantes asistentes a tres centros de AT de Santiago de Chile. Los resultados apuntan a concepciones definidas sobre la DI, actitudes que oscilan entre un distanciamiento temporal y un mayor grado de comprensión del fenómeno, sentimientos iniciales de frustración, pena, rabia e invisibilización personal, familiar y proyecciones profesionales asociadas a la DI.
\end{abstract}

Descriptores: Discapacidad intelectual; Primera infancia; Hermanos; Hermanas; Familia.

\begin{abstract}
The study falls within the scope of Early Care and Intervention. It was proposed to contribute to the approach of intellectual disability (ID), from a systemic-family perspective, stressing the contribution of socio-family agents in the construction of the phenomenon. In order to do so, he gave voice to the brothers and sisters of children with Down's syndrome, investigating their socio-cognitive representations (conceptions, attitudes and feelings) about themselves, his brothers and sisters in ID situation and his role in the family. The qualitative methodological design used indepth interviews oriented by thematic script as tools for collecting/producing information, based on family-centered approaches. The study group consisted of 18 Chilean brothers and sisters and 2 immigrants attending three stimulation and early care centers in Santiago of Chile. The results point to defined conceptions about ID, attitudes ranging from a temporary distance to a greater degree of understanding of the phenomenon, initial feelings of frustration, grief, rage and personal and family invisibilization and professional projections associated with ID.
\end{abstract}

Keywords: Intellectual disability; Early childhood; Brothers; Sisters; Family.

*Contacto: paola.andreucci@ugm.cl

ISSN: 0718-7378

www.rinace.net/rlei/
Recibido: 12/07/2019

$1^{a}$ Evaluación: 15/08/2019

Aceptado: 20/09/2019 


\section{Introducción ${ }^{1}$}

La perspectiva de la familia en temáticas de discapacidad, cuya condición afecta a alguno/a de sus integrantes, viene tomándose la agenda investigativa sólo desde los últimos años. Por ello, la relevancia temática de este estudio está asociada a la literatura científica reciente que ratifica que el apoyo a la familia y a sus integrantes resulta fundamental cuando se aborda la problemática de la discapacidad en tanto construcción social (Guralnik, 2016; McWilliam, 2016; Simón, Giné y Echeita, 2016) y se privilegia un enfoque de derechos humanos para la discapacidad. En esta dirección, se asume la convención de los derechos del niño (Lozano-Vicente, 2016; Luciani-Conde, 2010), entendida esta como una normativa regulatoria que protege los requerimientos de los niños, las niñas, los y las adolescentes, donde no solamente se explicitan los derechos con los que cuentan estos individuos, sino también las obligaciones del Estado y la familia de proveer orientación a los padres y madres y, desde ellos, a sus hijos/as para un claro ejercicio de sus derechos conforme a la evolución y potenciación de sus capacidades (Cecchini y Martínez, 2011; Palacios Rizzo y Bariffi, 2014).Lo anterior se plasma, entre otras aristas fundamentales del enfoque de derechos, en los principios y orientaciones de una atención temprana efectiva en la primera infancia, en contextos familiares o no familiares, inéditos o incluso inhabituales (Cara-Díaz, Sola-Martínez, Aznar-Díaz y Fernández-Martín, 2015; Palomares-Ruiz, Sánchez-Navalón y Garrote-Rojas, 2016).

En la experiencia internacional comparada, no obstante, no ha prevalecido una mirada integral, biopsicosocial, sistémica y/o ecológica acerca de la discapacidad, ya que el paradigma dominante desde el aspecto político, médico, social, educacional y familiar se ha centrado en la persona en situación de discapacidad (Alfaro, 2017) y, muy incipiente y secundariamente, en sus padres (Guralnick y Bennett, 1989; Guralnick, Connor y Johnson, 2011 ). Según las teorías sistémicas familiares, por su parte, lo que afecta a algún integrante de la familia con algún grado de discapacidad inevitablemente va a repercutir en el resto de sus miembros, ya sean aspectos positivos o negativos (Leal, 2008). Por consiguiente, tanto el foco en el niño o niña como en la familia son ambos igualmente importantes, debido a que, si desde la familia existe una transformación positiva en vías del crecimiento, ésta va a repercutir satisfactoriamente en el bienestar y desarrollo del niño o niña, y viceversa. Entre más centrado el trabajo en las familias a través de la capacitación, empoderamiento, atribución de autoridad y autogestión de sí mismas, más van a poder desarrollar sus fortalezas y recursos en las prácticas de crianza y en los cuidados cotidianos que todos/as sus integrantes requieren (Viloria y González, 2017). Esto significa que la familia se constituye en un eje fundamental y motor de cambio para cada uno de sus miembros, especialmente para aquellos eventualmente más vulnerables por cursar etapas tempranas de su desarrollo y/o por transitar o permanecer con algún grado de discapacidad (Simón, Giné y Echeita, 2016).

Existe una cada vez más amplia literatura que habla sobre enfoques centrados en la familia cuando se aborda la situación de discapacidad en atención temprana (Guralnick, 2011, 2016), no obstante, tiende a olvidar que la familia consta de más integrantes. Los hermanos

${ }^{1}$ Este estudio se enmarca en una investigación más amplia titulada "Evaluación de la atención temprana de un grupo de niños y niñas con Síndrome de Down y sus familias por parte de las áreas de Educación y Salud de la Universidad Ucinf” (Proyecto acogido al Art. ${ }^{\circ} 69$ de la Ley 18681) implementado entre el o1 de enero de 2016 y el 31 de diciembre de 2016 , con apoyo del Ministerio de Educación de Chile (MINEDUC). 
y las hermanas, requieren sortear diferentes problemáticas en el curso del desarrollo y, generalmente, son dejados de lado por privilegiar la atención en el hijo o hija con algún grado de discapacidad (Iriarte y Ibarrola-García, 2016). Cabe señalar que el individuo se construye desde su participación e interacción con la familia y la sociedad, es decir, tanto el sentido de pertenencia como el de separación-individuación se logra en la interacción familiar y extra-familiar (Minuchin, Nichols y Lee, 2007). La individuación resulta fundamental para lograr sujetos activos, autónomos, integrados y que resulten en un aporte a una sociedad en desarrollo. Incluir, por tanto, la dimensión fraternal permitiría relevar la individuación como una resultante de la interacción entre pares y no sólo como un producto de las prácticas de crianza de los padres, madres y de la intervención de los/as profesionales.

Por su parte, los hermanos y hermanas de personas con discapacidad necesitarían, en primera instancia, tener información real de lo que ocurre, desarrollar e implementar herramientas para responder a los requerimientos familiares y societales, así como también expresar sus sentimientos validando el ejercicio de sus concepciones y emociones respecto a la situación de discapacidad de su hermano/a y el impacto familiar que conlleva (Lizasoain y Onieva, 2010).

Este estudio pretende acceder a las necesidades de los hermanos y hermanas, conocer sus vivencias y sentimientos más prevalentes, comprendiendo la representación que tienen de su lugar en la familia, de su rol en la relación con su hermano/a en situación de discapacidad y sus necesidades socio-educativas. Lo anterior a objeto de contribuir a evidenciar su posición subjetiva y a su vez ser un aporte en la detección temprana de eventuales desajustes emocionales en ellos/as con diversas implicancias en su proyecto de vida, proceso educativo e instancias de integración psicosocial. Por ello, nos preguntamos: ¿Cuáles son las concepciones, actitudes y sentimientos de hermanos y hermanas de niños y niñas en situación de discapacidad intelectual, en relación a sí mismos, su/s hermano/as y su rol en la familia?, ¿Cómo esta develación, desde el protagonismo de los/as hermanos/as, permite comprender el fenómeno de la discapacidad intelectual como una problemática sistémica compleja y culturalmente situada?

\section{Marco de referencia}

Este marco conceptual aborda la problemática de la discapacidad intelectual desde las propuestas de la Atención e Intervención Tempranas, por una parte, y los enfoques centrados en la familia, como enfoques que relevan los derechos y deberes de los/as ciudadanos/as y potencian las subjetividades de sus integrantes en una comprensión social de la discapacidad, por otra. En este último punto se incorpora una revisión específica sobre las necesidades y percepciones de los/as hermanos/as de las personas con Síndrome de Down, considerando que son ellos (as) quienes han adquirido un rol dentro de la red de cuidados y se constituyen en figuras clave en el proceso de intervención dado el nivel de apego que tienen con sus hermanos (as) (Perpiñán, 2011). Lo anterior, en algún grado, ha incidido en su propia construcción, experiencias y narrativas, y por tanto, en sus significaciones del mundo.

\subsection{Discapacidad Intelectual (DI) y Atención Temprana (AT)}

La Asociación Americana de Deficiencia Intelectual (AAIDD) señala como concepto de DI "una discapacidad caracterizada por limitaciones significativas en el funcionamiento 
intelectual y la conducta adaptativa, tal como se ha manifestado en habilidades prácticas, sociales y conceptuales. Esta discapacidad comienza antes de los 18 años" (MINEDUC, 2007, p. 7). En este sentido, el término Discapacidad Intelectual es usado cuando una persona presenta limitaciones en las habilidades intelectuales de razonamiento, planificación, solución de problemas, pensamiento abstracto, comprensión, rapidez en el aprendizaje, entre otras. A lo anterior se suma un conjunto de habilidades conceptuales, sociales y prácticas, que son de vital importancia en la vida cotidiana (MINEDUC, 2007).

La DI es un concepto complejo y multifactorial. Se ha abordado, hasta la fecha, desde cuatro modelos o perspectivas diferentes (Palacios Rizzo y Barifi, 2014): (1) Modelo de prescindencia, asocia la discapacidad a motivos religiosos, cuya anormalidad responde a mensajes diabólicos perpetrados por dioses enojados y castigadores de ciertas personas y prácticas humanas. (2) Modelo médico o rehabilitador, enfatiza las dificultades y limitaciones de la persona en situación de discapacidad proponiéndose normalizarla para su integración a la sociedad; el problema es de la persona y, eventualmente, de su entorno inmediato, pero no de la sociedad en su conjunto. (3) Modelo social, relaciona la discapacidad a causas sociales interpelando a una sociedad cuya preparación es insuficiente y obstaculizante para incluir la diversidad de requerimientos de sus ciudadanos/as, proponiendo focalizarse en las fortalezas y potencialidades de las personas más que en el ejercicio de normalización propuesto por el modelo médico (Martínez, 2011). (4) Modelo biopsicosocial, se representa la discapacidad como una consecuencia de la interacción entre factores biológicos, psicológicos y las características del contexto socio-cultural en que se inserta la persona en situación de discapacidad (Turner, 2001). Desde esta perspectiva, la respuesta a las necesidades y las intervenciones que deben realizarse, requieren centrarse en la relación persona-ambiente, potenciando los recursos individuales y los del contexto de carácter con el fin de aportar a un ambiente inclusivo (Peñas-Felizzola, 2013).

Desde este último enfoque y extremando el componente social del modelo que aporta a la generación de ambientes más inclusivos y una clara atención a la diversidad, se asume en este artículo una concepción de "la discapacidad (como) una construcción sociocultural e histórica, que impacta a las personas en situación de discapacidad y a sus cuidadores" (Giaconi, Pedrero y San Martin, 2017, p. 55), asimismo, se construye fundamentalmente en el núcleo familiar y tributa a sus estructura, dinámica, reglas, roles, posicionamientos $\mathrm{y}$ funciones al interior de la familia.

Este tipo de enfoque ha sido integrado por el ámbito de la atención e intervención tempranas. La AT se ha concebido como un pilar para el logro de los procesos de aprendizaje y la integración de niños, niñas y jóvenes con distintos tipos y grados de discapacidad en la educación y en la vida activa de sus sociedades de pertenencia y/o referencia (Guralnik, 2011). Se entiende por AT:

(al) conjunto de intervenciones dirigidas a la población infantil de 0-6 años, a la familia y al entorno, que tienen por objetivo dar respuesta lo más pronto posible a las necesidades transitorias o permanentes que presentan los niños con trastornos en su desarrollo o que tienen el riesgo de padecerlos. Estas intervenciones, que deben considerar la globalidad del niño, han de ser planificadas por un equipo de profesionales de orientación interdisciplinar (Federación Estatal de Asociaciones de Profesionales de Atención Temprana, 2000, p.10)

Haciendo un poco de historia con el propósito de situar la AT en la actualidad, en los años 60 en Estados Unidos comenzaban a ejecutarse los primeros programas de estimulación 
temprana, los cuales estaban destinados específicamente a atender las necesidades de niños y niñas en situación de vulnerabilidad socioeconómica importante. Luego, este escenario fue mutando a la atención de niños y niñas que presentaban alguna discapacidad, lo cual se reguló en el año 1986 con la creación de una ley que aporta a la definición de los derechos de la familia y creación de planes de AT en ese país. (Salar, 1993, citado por Sánchez, 2015).

En Europa, específicamente en España, la AT emerge como concepto y práctica en la década de los 70, con una perspectiva en la atención de rehabilitación, por lo cual estaba centrada en el trastorno o alteración propiamente tal. Este enfoque comenzó a cambiar gradualmente, siendo un hito importante en este cambio de paradigma hacia la inclusión de la familia, la publicación del Libro Blanco de AT creado por la GAT en el año 2000. (Escorsia-Mora et al., 2018).

Respecto a los albores de la AT en Chile. "(...) desde el año 1978 se inicia un programa piloto sobre estimulacion precoz, lo que derivó en los consultorios, evaluaciones en el control del 'Niño Sano' y estimulación del desarrollo psicomotor para los que presentaban deficiencia en esa área" (United Nations International Children's Emergency Fund; UNICEF, 2007, citado por Pérez Lisboa, 2019, p. 106). El enfoque del programa como ha ocurrido en otras latitudes, era fundamentalmente biomédico y rehabilitador. La derivación a programas de corte psicosocial con consideración de la familia, puede sondearse hasta hace algunos años atrás (2007), con la puesta en marcha del "Chile Crece Contigo" como Subsistema de Protección Integral a la Infancia. Dicho subsistema, dependiente del Ministerio de Desarrollo Social, considera fundamental el trabajo en red con el ámbito municipal, sistema de salud, educación y otros entes de la comunidad como la familia y su entorno directo (Saavedra, 2015).

En el vasto espectro de las discapacidades de las que se ocupa el dispositivo de AT y sus equipos multidisciplinarios se encuentran las discapacidades mentales, intelectuales o cognitivas y, específicamente, el Síndrome de Down. El libro blanco de la Atención Temprana (GAT, 2000) se plantea como objetivos, para estos niños y niñas: (1) Reducir al máximo los efectos de una deficiencia sobre el desarrollo global del niño o de la niña; (2) Considerar al niño y a la niña como sujetos activos de la intervención; (3) Adaptar el entorno a las necesidades específicas del niño y/o la niña; (4) Atender las necesidades de la familia que derivan de la situación de tener que atender, a su vez, a las necesidades médicas y educativas de un niño o niña en alto riesgo.

Desde hace varias décadas, se ha sabido que la intervención multidisciplinaria en AT y la implicación de profesionales y familia en el abordaje precoz de los niños y niñas con alto riesgo de deficiencias y necesidades educativas especiales, cambia el pronóstico y las perspectivas de desarrollo de estas poblaciones, incluso en los casos de afectaciones anatómicas muy severas (Arrabal y Arizcun, 2005; Ponte, 2017; Veerman, Cuevas y Arizcun-Pineda, 2005). Esta aproximación, no obstante, no ha ocupado un lugar relevante sino hasta muy entrado el siglo Xx (Guralnick y Bennett, 1989).

\subsection{Enfoques centrados en la familia}

En las dos últimas décadas, en esta materia, se consolida el estudio y la investigación de la infancia temprana (0-4 años), se producen avances teóricos y conceptuales con el desarrollo de un marco común de referencia, se logra apoyo científico sobre la efectividad de los programas y se obtienen consensos en los principios marco para el diseño e 
implementación de sistemas de AT. Junto a ello, se produce un gran y renovado impacto en la generación de políticas públicas en variados países del mundo desarrollándose diversos tipos de programas de intervención infantil, prácticas basadas en evidencia científica y de experiencias comparadas, diseño e implementación de sistemas de AT con focos formativos, de derechos (Hernández, 2015) interventivos y sociales cuya efectividad se logra evaluar sistemáticamente (Guralnick 2016; Vegas et al., 2006). Los autores son bastante claros en señalar la importancia de la familia, de padres y madres, en su rol de cuidadores/as y formadores/as durante todo este proceso (García-Sánchez et al., 2014).

Este giro de la AT hacia el protagonismo de las familias responde a lo que se ha empezado a conocer como «enfoques centrados en la familia» (Álvarez, Alonso y Amor, 2016; McWillam, 2016) y que tienen como propósito la consideración de los ámbitos naturales de inserción y tránsito del niño o niña, es decir, del contexto hogar- escuela (Simón, Giné, y Echeita, 2016) en el abordaje de la discapacidad y la inclusión de los padres, madres y cuidadores/as con sus respectivas prácticas de crianza como agentes fundamentales en los procesos de desarrollo y aprendizaje de sus hijos e hijas impactando, a su vez, en la propia organización familiar en tanto estructura, dinámica, reglas, roles y sistemas de apego (Bowlby, 1993; Flores y Porges, 2017; González, Blanco y Herrero, 2017; PalaciosHernández, 2016; Viloria y González, 2017). Reconocer, por tanto, el aporte y rol protagónico de los hermanos y hermanas de niños y niñas en situación de DI, validando sus puntos de vista y legitimando sus sentimientos, destaca como una tarea pendiente de este tipo de enfoques.

\section{Percepciones y necesidades de hermanos/as de niños/as con Síndrome de Down}

$\mathrm{El}$ arribo de un/a nuevo/a integrante con algún grado de discapacidad remueve al sistema familiar. Este tiende a auto regularse paulatinamente retornando a su homeostasis o generando una nueva conformación (Arellano y Peralta, 2013). La reorganización o nueva conformación incluye la adquisición de los roles y funciones que asumen los/as hermanos/as. De acuerdo con Ruiz Aparicio y Tárraga Mínguez (2015), los/as hermanos/as mayores son considerados fuentes de apoyo a nivel emocional, modelos de identificación y personas con las que se compartirán las primeras interacciones comunicacionales, estimuladores naturales para un/a niño/a en situación de discapacidad. A esto se añade que en muchas ocasiones deben tomar roles de cuidado más complejos.

Por otro lado, se generan sentimientos negativos acerca del/de la hermano/a en situación de discapacidad, según Núñez (2007, citado en Mirabal, 2015), quien observó la generación de sentimientos tales como la culpa por ser el sano, a la vez que asumen la responsabilidad de reparar aquello que los padres y madres sufren por ese/a hermano/a, dándoles alegrías. Por otra parte, experimentan rivalidad por la poca atención prestada, ya que los padres y madres estarían preocupados del/de la hermano/a en situación de discapacidad, y bastante frustración en la relación que se genera, pues la visualizan como diferente a las demás. Aun así, son capaces de alegrarse por sus logros, manifestando amor y/o ternura por él o ella, y en muchas ocasiones desarrollan temor respecto de lo que vaya a suceder en el futuro.

En el marco de este ámbito más descuidado de los enfoques centrados en la familia en AT, la fratría, nos propusimos como objetivo del estudio describir las necesidades de los hermanos y hermanas, y conocer sus vivencias y sentimientos más prevalentes. Secundaria y complementariamente, nos interesó (1) comprender la representación que tienen de su 
lugar en la familia, de su rol en la relación con su hermano/a en situación de discapacidad y (2) acceder a sus propias necesidades socio-educativas.

\section{Método}

El estudio, inmerso en los enfoques cualitativos (González-Monteagudo y Ochoa-Palomo, 2014; Sarasa, 2014) recoge las narrativas a través de las siguientes técnicas: relatos de vida (Aguilar y Chávez, 2013; Peregrina et al., 2015) los que se llevaron a cabo bajo la técnica de entrevista abierta (Schriewer y Agea, 2015), una modalidad específica de entrevista en profundidad, rescatando la trayectoria socio-emocional de los sujetos en estudio -es decir su tiempo recorrido-, su pasado y presente personal, incluidas concepciones, necesidades, sentimientos, logros, frustraciones y expectativas. Se abordan las entrevistas en profundidad, según guión o preguntas-guía, que exploran en las dimensiones de familia y contex to de procedencia, recuerdos de las distintas etapas del desarrollo y escolares, arribo a la educación terciaria (si corresponde), imágenes del desarrollo personal actual y proyecciones. La técnica elegida permite un compromiso con la búsqueda de la comprensión y la cercanía a los sujetos en una perspectiva de «insider» (Kornblit, 2004). Lo relevante de este enfoque, es que nos permite hacer un análisis de aquellos aspectos comunes en cada relato, para así comprender cómo se construyen identidades, experiencias y significaciones, considerando el papel de la cultura y de la sociedad, y a su vez, cómo se conjuga lo público y lo privado en dichas narrativas.

Los/as participantes fueron contactados a través de sus familias asistentes a tres centros de estimulación, atención y/o intervención tempranas adscritas a la Red de AT-Chile. Los tres centros pertenecen a instituciones de educación superior privada de Santiago de Chile y han avanzado desde modelos biopsicosociales hacia modelos centrado en la familia. En términos generales, por lo tanto, los tres centros se orientan por enfoques de derechos y enfoques centrados en la familia.

Ahora bien, respecto de los criterios de selección fueron los siguientes: tener entre $17 \mathrm{y}$ 25 años de edad, vivir en la misma casa que su hermano (a) y tener algún rol específico en lo que respecta al cuidado. Accedieron a participar 25 familias y completaron las entrevistas bajo consentimiento informado y asentimiento informado, según sea el caso, 20 hermanos/as entre 17 y 23 años, con un promedio de 18,7 años, 18 de procedencia chilena y 2 de procedencia colombiana, 15 mujeres y 5 hombres.

Dentro de las perspectivas analíticas planteadas por Riessman (2008) se utilizó el denominado análisis temático, cuyo foco está en el contenido del relato y su relación con recursos lingüísticos y culturales disponibles en esa sociedad o culturas (lo “dicho"). El levantamiento semántico de las categorías se realizó con el apoyo del software de análisis cualitativo ATLAS TI $7.0{ }^{\circledR}$

Respecto de los aspectos éticos, se entregó formato de consentimiento informado a todos los(as) participantes adultos, asentimiento informado a los menores de edad y consentimiento informado a sus padres y/o madres. Allí se explicitan las características del estudio, el modo en el que se considerará su participación, sus principales beneficios y riesgos. Se cauteló la confidencialidad del relato hacia los padres y madres toda vez que los/as participantes así lo solicitaron.

Las entrevistas fueron realizadas por las investigadoras del estudio en los centros AT, con posterioridad a las atenciones habituales estipuladas para esa semana, y la transcripción 
ortográfica de las mismas fue realizada por la asistente de investigación. Las entrevistas se extendieron por un período de tiempo de 42 a 58 minutos. La codificación se realizó alternadamente y se sometió a validación interjueces los extractos seleccionados para cada categoría.

\section{Resultados}

Los resultados se presentan en una figura con los códigos relevantes que emergieron de las entrevistas realizadas, en primer lugar y, luego de ello, se da paso al análisis de cada categoría o anudamiento de códigos que para efectos de este artículo hemos denominado “dimensión”, en términos descriptivos e interpretativos, basado e ilustrado en la evidencia de las citas textuales de los/as entrevistados/as. Se espera que las dimensiones seleccionadas con apoyo de ATLAS TI 7.0 ( , cuyos extractos referenciales fueron debidamente validados por el procedimiento de interjueces, den respuesta a las preguntas y objetivos del presente estudio.

En la figura 1 se muestra la codificación focalizada, en donde a modo de síntesis se puede observar cómo se configura la percepción de hermanos y hermanas en torno a la DI en relación con otros aspectos referidos a concepciones, vivencias y elementos de índole personal.

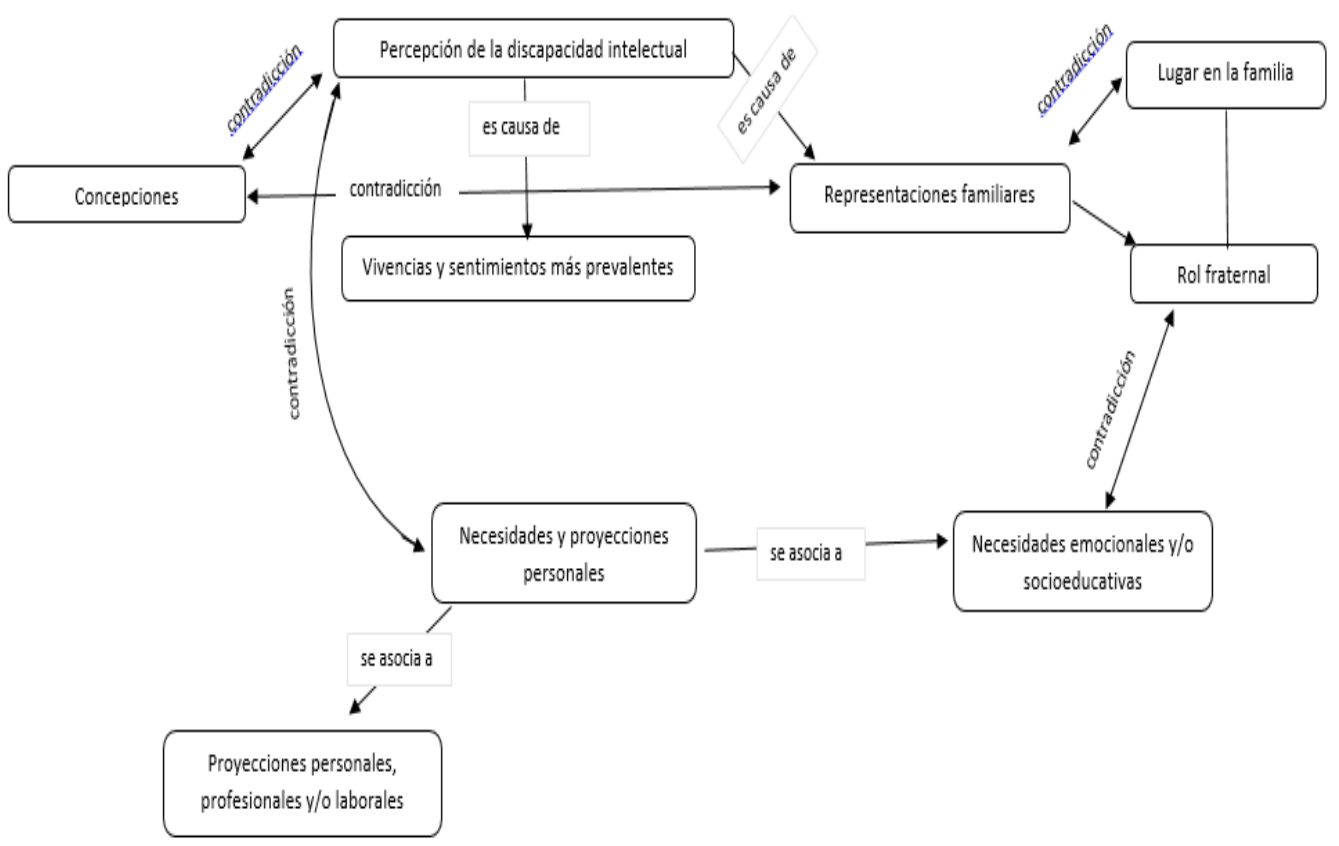

Figura 1. Codificación focalizada percepción hermanos/as en torno a la DI Fuente: Elaboración propia.

Los hermanos y hermanas reflexionan en torno a cómo dicha percepción está en constante contradicción respecto de las concepciones y cómo ello impacta, en alguna medida, a las representaciones familiares en conjunto con las respectivas dinámicas que se viven en su interior. A su vez, dicha percepción en torno a la DI, está atravesada por vivencias y sentimientos generados a raíz de la discapacidad intelectual. Por otro lado, en contraste, se ubican las necesidades y proyecciones más personales, desde las cuales se desprenden las necesidades emocionales y/o socioeducativas estrechamente vinculadas al lugar y rol 
que se ocupa en el espacio familiar. Para, finalmente, reflexionar en torno a sus proyecciones personales, profesionales y/o laborales.

\subsection{Percepción de la discapacidad intelectual}

En esta dimensión se incorporan las concepciones que los hermanos y hermanas han elaborado en relación a la discapacidad intelectual y las vivencias, sentimientos y emociones asociadas a esta concepción.

\subsubsection{Concepciones}

Tiende a predominar una visión de la discapacidad como una condición "diferente" sin focalizarse en sus limitaciones o barreras sino en sus fortalezas y potencialidades. Se enfatiza, al mismo tiempo, que la discapacidad tiende a visualizarse como algo extraño fuera del núcleo familiar mientras que bastante habitual o "natural" desde dentro de la familia.

Nunca he percibido que [mi hermano] sea diferente al resto... desde muy chico lo hemos tratado como un miembro más de la familia...para mí, no hay diferencias y mis papás tampoco las han hecho nunca. (hombre, 17 años, chileno)

Para todos ha sido difícil adaptarse al país... también para [mi hermana]...pero nosotros la tratamos igual que a todos...ella vino por nuevas oportunidades como toda la familia...en el centro la tratan como una más...quizás en la calle la miran con pena o rehúyen la mirada. (mujer, 23 años, colombiana)

Me quedo con la idea de las «capacidades diferentes $>$...ella no es una discapacitada...tiene una serie de virtudes que yo no tengo; es muy perceptiva, sensible y afectuosa...empática, diría yo...me ha enseñado mucho, siendo tan pequeña aún. (mujer, 20 años, chilena)

La han estimulado bastante desde recién nacida...tanto en la casa como en el centro...esa es la única diferencia que veo con los otros niños... mi mamá también lo hizo conmigo, pero con [mi hermana] ha sido más y más constante. (mujer, 19 años, chilena)

\subsubsection{Vivencias y sentimientos más prevalentes}

Predominan sentimientos negativos de pena, rabia e indignación en relación a la percepción de la discapacidad por parte de la sociedad, al mismo tiempo que orgullo por cómo sus familias han enfrentado las eventuales dificultades asociadas a la DI.

\footnotetext{
Me da rabia la ignorancia que tiene la gente frente a este tipo de niños distintos... si ven a un niño en silla de ruedas como que entienden más... al ver a mi hermana, se sienten como incómodos, la miran con lástima... me indigna su actitud. (mujer, 20 años, chilena)

Pena....me da mucha pena la actitud de la gente... se acuerdan para las campañas y se olvidan en el día a día... sin el apoyo de este tipo de centros y de la fuerza que ha tenido mi familia, nos habría costado salir adelante... me siento muy orgullosa de mis padres. (mujer, 18 años, chilena)

Cada día aprendo algo distinto de él...espero verlo crecer, desarrollarse y ser feliz...mi mami dice que es como un angelito y yo lo creo así...me siento feliz de tenerlo como hermano... y me da pena la gente que no tiene idea de nada... jincluso piensan que estos niños se mueren de guaguas! (hombre, 21 años, chileno)
}

\subsection{Representaciones familiares}

En esta dimensión se incluyen las representaciones que tienen de su lugar en la familia y de su rol en la relación con su hermano/a en situación de discapacidad intelectual. En esta 
última categoría se aprecian modificaciones a lo largo de las etapas del desarrollo y una visión particular desde el proceso adolescente que están transitando.

\title{
3.2.1. Lugar en la familia
}

Se asume una posición secundaria en la familia y un cierto grado de invisibilización respecto de la presencia y lugar ocupado por su hermano/a con DI. Al mismo tiempo, se argumenta en función de la carga asumida por sus padres y madres y la conmoción familiar que produjo el nacimiento del/de la hermano/a, cuestión que ha ido cambiando con los años.

\begin{abstract}
Al principio todo se volcó en él...es lógico... recuerdo que me dejaban en casa de mi tía o de amigos mientras se organizaban... me sentí algo desplazado...como que sobraba. Ahora he asumido el rol de hermano mayor; lo acompaño a los controles y al centro, muchas veces... me siento aportando a mi familia. (hombre,2 1 años, chileno)

Cuando más chica no lo podía entender...estábamos tan bien los tres...yo nunca pedí un hermanito o hermanita...y de repente salió... lo sentí como un castigo y estuve harto tiempo enojada y triste... mi vida cambiaba... ya nadie me ponía atención... si me enfermaba o tenía un problema en el colegio o con amigas, siempre era algo menor en comparación al tema de mi hermana... incluso pensé en desaparecer... (mujer, 19 años, chilena)

Mi tío, el hermano de mi papá, fue fundamental pa mí en este proceso...él es mi tío, pero es joven, él me escuchaba y podíamos conversar temas que no hablaba con mis papás...no me atrevía o no los quería cargar más con problemas sin importancia... hasta hoy en día somos amigos... ahora hablamos de minas y esas cosas (jajaja). (hombre,20 años, chileno)
\end{abstract}

\subsubsection{Rol fraternal}

En esta categoría se aprecian diferencias culturales en la representación del rol fraternal y sus implicancias para el desarrollo de su hermanos/as y su familia. En ocasiones, tiende a confundirse y tornarse ambiguo el rol (sobre) protector, atribuyéndose funciones en la crianza y la contención emocional más propios de la diada parental. En otras, se percibe el rol como complementario al rol de los padres.

\footnotetext{
Ahora la veo como mi hermana menor y la cuido mucho...ella es bien linda, pretensiosa y también le enseño cosas de mujeres... la única lata es cuando se mete en mi pieza y en mis cosas...cuando la vi con mi polera favorita, la reté... jaunque se veía muy divertida! (mujer, 19 años, chilena)

Mi hermana ha sido mi hermana desde que nació... si Diosito lo quiso así y nos envió esta angelita es para cuidarla y acompañarla siempre... ella estará con mi familia hasta que muera e incluso les hará compañía a los padres cuando estén ancianos...como hermana mayor debo hacerme cargo y ya lo estoy haciendo con mi trabajo en este país. (mujer, 20 años, colombiana)

Ayudo a mis padres en lo que puedo...el que estemos juntos como familia es lo más importante para mi... me siento responsable de eso... a veces, echo de menos carretear más y hacer otras cosas con amigos... mis fines de semana son todos en familia y todos nos ocupamos de [mi hermano]...nos rotamos. (hombre, 21 años, chileno)
}

\subsection{Necesidades y proyecciones personales}

En esta dimensión se articulan las categorías de necesidades emocionales y socioeducativas de los hermanos y hermanas de niños y niñas en situación de discapacidad intelectual y sus proyecciones personales, profesionales y/o laborales. 


\title{
3.3.1. Necesidades emocionales y socioeducativas
}

En esta categoría persiste cierto grado de invisibilización familiar respecto a las necesidades emocionales y socio-educativas de los/as hermanos/as de niños/as en situación de discapacidad intelectual y una búsqueda de satisfacción o complemento por vías alternativas (familia extendida, compañeros de curso, amistades).

\begin{abstract}
Le contaba mis cosas a mi tía y ya de más grande a mi tío y mis amigos...no quería molestar a mis papás con mis cosas... a ellos les ha tocado duro...no creo se hayan enterado de muchas cosas de mi cuando fui creciendo, si hasta antes que nació [mi hermano], después, hubo un período en blanco... los amigos me ayudaron harto, son mis yuntas hasta ahora. (hombre, 21 años, chileno)
\end{abstract}

Nunca he sido muy comunicativa...como que me aislé más con el nacimiento de mi hermana...obvio que hay un cambio, un shock al principio... yo era bien apegada a mi mami...como mamona...jajaja... lo debo haber resentido, lógico... ahora somos como amigas, las dos bajo la causa de sacar adelante a [mi hermana]. (mujer, 19 años, chilena)

Mis amigas son mis confidentes...nos contamos todo... en esta edad ya no se necesita tanto a la mamá... ya hay otros temas e intereses... igual no se los contaría a mi mamá, aunque estuviera más disponible para escucharme... iya estoy grande! (mujer, 20 años, chilena)

\subsubsection{Proyecciones personales, profesionales y/o laborales}

En esta categoría se abordan las expectativas personales y profesionales de los/as hermanos/as de niños/as en situación de DI. Destaca la elección por carreras profesionales u ocupaciones de servicio social y de corte humanista o socio-educativo, con cierto desdén por los trabajos vinculados al ámbito de la medicina tradicional. Por otra parte, algunos/as hermanos/as no logran proyectarse aún, permaneciendo su vida algo "estancada”.

No me dedicaría nunca a algo médico... los médicos son muy brutos... imagínese que le dijeron a mi mami en el parto que [mi hermano] venía con Síndrome de Down... y ven sólo problemas, diagnósticos y limitaciones en las personas diferentes... si no hubiera sido por este centro (CET) estaríamos muy mal como familia... sin esperanza y futuro para mi hermano...y para todos. (mujer, 18 años, chilena)

Estoy estudiando Educación Diferencial...es mi vocación...claro que todo tiene que ver con mi hermana...necesito ayudar a estos niños, sentirme útil y aportar con mi experiencia personal... si miro hacia atrás era otra mi elección [Diseño Gráfico], pero [mi hermana] vino a cambiarlo todo, incluso mis prioridades...lo único que quiero es ayudar. (mujer, 20 años, chilena)

No tengo idea qué va a ser de mi vida más adelante... he estado dando bote de carrera en carrera... ahora pienso en algo técnico, rápido, que gane plata luego para aportar a mi familia y darme algunos gustos... me he sentido sin rumbo...en blanco... paralizado por harto rato. (hombre, 22 años, chileno)

Vinimos buscando mejor trabajo y oportunidades y en eso estamos...luchando... luchando todos como familia...por ahora hago turnos en un colegio...y trabajo en otros dos...me gusta el asunto educacional, espero poder estudiar más y dedicarme seriamente a eso... ando bien cansada, pero debemos salir adelante. (mujer,23 años, colombiana)

(...) por eso decidí estudiar Psicología... si hubieran tenido más psicología... más empatía con nosotros como familia, creo nos habría costado menos salir adelante...muchos temas asociados a la discapacidad habrían sido comprendidos antes, incluso, con mayor información, pero de una forma cercana, sensible, a escala humana. (mujer, 19 años, chilena) 


\section{Discusión y conclusiones}

Los derechos de las personas con discapacidad (ONU, 2016) suponen considerar sus fortalezas y potencialidades y, al mismo tiempo, derribar las barreras físicas y psicosociales que les impone el entorno y que limitan su desarrollo. La desestimación del aporte de la familia y de sus integrantes puede resultar en un atentado contra sus derechos, toda vez que el fenómeno de la discapacidad se construye y sitúa en una determinada realidad familiar y sociocultural. En este sentido, resulta necesario poner atención a la unidad familiar y sus respectivos subsistemas (padre, madre, hermano/a, etc.), los cuales se relacionan entre sí y/o con otros elementos-contextos afectando de manera positiva o negativa al sistema en su totalidad (Aparicio y Tárraga, 2015). Bajo esta perspectiva, la presencia de una persona en situación de discapacidad, modifica no sólo las dinámicas, sino que además al propio entorno familiar (Valverde, 2017).

Desde esta óptica es que se han tomado en consideración, las concepciones, vivencias y sentimientos de los/as hermanos/as de niños/as con DI en relación a la discapacidad intelectual, constatando una percepción bastante clara respecto a la situación de menoscabo impuesto por las condiciones sociales y culturales frente a la discapacidad más que una visión esencialista del fenómeno aislado al sujeto y sus falencias de nacimiento. Asimismo, se precisa el valor diferencial otorgado a la discapacidad al interior de la familia vs las percepciones que se reciben desde el medio. Coadyuvantes a una percepción favorable de la DI, centrada en los recursos y las potencialidades más que en sus limitaciones, se releva el trabajo realizado por los centros de estimulación e intervención tempranas a los que asisten los/as niños/as y participa activamente toda la familia.

Allí, radica la importancia de un abordaje que considere la perspectiva de hermanos y hermanas de niños (as) con Síndrome de Down, puesto que:

(...) puede llegar a ser un vínculo afectivo muy significativo y duradero para muchas
personas. Los hermanos son una fuente importante de apoyo a nivel emocional, ejercen
muchas veces como modelo de identificación y representan una de las primeras fuentes
de interacción social para los niños. (Aparicio y Tárraga, 2015, p. 190)

La misma polarización anterior- endogámica vs, exogámica- se aprecia a la hora de explorar las vivencias y sentimientos más prevalentes, al predominar emociones y sentimientos negativos de tristeza, enojo e indignación frente a la percepción "ignorante" de la DI por parte de la sociedad, mientras se enaltecen casi reivindicativamente, el honor, la fuerza y el orgullo frente a sus familias que han sabido salir adelante frente a la adversidad. Esta dicotomización también puede responder a la etapa del desarrollo que cursan los/as jóvenes entrevistados/as. Si bien,los estudios han enfatizado que los/as hermanos/as de personas en situación de discapacidad intelectual "pueden presentar problemas de identificación y socialización, necesidad de compensación, adopción temprana de grandes responsabilidades, sentimientos de abandono, culpa, vergüenza o tristeza" (Lizasoain y Onieva, 2010, p. 89), no se habían referido con claridad a los sentimientos positivos y/o compensatorios expresados por los/as hermanos/as de niños/as en situación de DI, asociados al rol y función de la familia en el proceso de integración de la discapacidad de uno de sus miembros. Lo anterior, probablemente estaría asociado a una cierta desestimación o inclusión incipiente de este tipo de enfoques sistémico-familiares en la investigación de la última década.

La condición de adolescentes de los/as hermanos/as entrevistados/as se torna más relevante al explorar las representaciones familiares, el lugar y rol que ocupan en la familia 
y ante sus hermanos(as). Varios/as de los/as hermanos/as consultados/as refieren un antes y un después en relación a su posicionamiento familiar. Desde una irrupción del/de la hermano/a con DI en una etapa de pre-pubertad o pubertad en que ellos/as ocupaban un lugar central en sus familias al concentrar la atención de sus padres dada sus primogenitura y condición de "hijos/as únicos/as" hasta el nacimiento de sus hermanos/as, hacia una rápida e imprevista invisibilización de sus requerimientos, planteamientos, sentimientos y necesidades de reconocimiento. Si bien, tienden a racionalizar a partir de argumentaciones claras y exculpatorias de sus padres, se aprecian dejos de frustración y cierta dificultad para asumir el nuevo rol: desde la condición unigénita, la primogenitura a la fratría. El rol fraternal, asimismo, es vivido con altas dosis de sobreprotección asociadas a la diferencia etaria y, en ocasiones, en complementariedad al rol paternal. En este ámbito, se aprecian diferencias culturales y religiosas que se integran al rol en una acepción más determinista («si Diosito lo quiso así y nos envió esta angelita...») y deontológica («como hermana mayor debo hacerme cargo y ya lo estoy haciendo con mi trabajo en este país»). En términos generales, su forma de relacionarse varía según una serie elementos, tales como: cantidad de hermanos (as), edades, género, origen, entre otras (Perpiñán, 2011).

Ahora bien, respecto del tipo de relación y la edad de cada uno (a) de los que forman parte de la fratría, Sonsoles Perpiñán (2011, p. 124) sostiene que:

\begin{abstract}
Generalmente, si son de edades próximas, son una fuente extraordinaria de estimulación. Interactúan con su hermano continuamente y además lo hacen sin ninguna carga de ansiedad. Juegan con él, le hablan, le quitan los juguetes, etc. En la lucha propia por sus derechos exigen al niño y a los padres, actitudes de normalización. Si son mayores puede que compartan emociones y roles de cuidador con la madre. Su relación con el hermano va a depender mucho del enfoque de los padres y de la información de la que dispongan. También están afectados por el tiempo disponible de los padres para ellos. En algunos casos pueden producirse situaciones de cierto abandono de los hermanos ya que el hijo con discapacidad reclama mucho tiempo y atención. Estos pueden resentirse desde el punto de vista emocional e incluso desarrollar síntomas para reclamar la atención de sus padres.
\end{abstract}

En el ámbito de las necesidades y proyecciones personales, se aprecian correlatos emocionales asociados al aislamiento y búsqueda de apoyo externo al núcleo familiar. Las amistades aparecen como las confidentes y "yuntas", sostenedoras emocionales del tránsito evolutivo de los/as hermanos/as de niños/as con DI, en estos últimos cuatro años de su desarrollo personal. Al irse convirtiendo en adolescentes los temas identitarios, centrales en este período, se habrían ido tramitando con el grupo de pares y a través de figuras significativas de la familia extendida ( «Mi tío, el hermano de mi papá, fue fundamental pa mí en este proceso...»). Al irse transformando en hombres y mujeres fuera de su núcleo familiar, en esta particular etapa del desarrollo, pudo haber contribuido al logro de la autonomía, la búsqueda de propósitos personales y a un proceso de mayor diferenciación del núcleo familiar.

Uno de los ámbitos más informativos lo constituyó la dimensión de las proyecciones personales, profesionales y laborales. Llama la atención la búsqueda compensatoria de actividades curriculares que les permitan enfrentar dificultades similares a las vividas en el núcleo familiar, específicamente con sus hermanos/as en su situación de discapacidad, desde una perspectiva profesionalizante. Se insiste en proveerse del conocimiento actualizado, oportuno y pertinente y de las herramientas más efectivas para abordar la problemática de la discapacidad intelectual desde los procesos formativos, la educación 
diferencial, la psicología y las ciencias humanas, situando a la familia en el centro de la (pre)ocupación de las intervenciones planificadas e implementadas. Lo anterior concuerda con los propósitos y lineamientos planteados por los enfoques de Atención Temprana centrados en la familia (Andreucci, 2016; García-Sánchez et al., 2014). El rechazo al enfoque médico, por su parte, hace referencia a su "brutalidad" en la aproximación diagnóstica que deshumaniza al individuo. Lo anterior, se suma a las consecuencias de este tipo de enfoque y de una concepción social que visualiza con temor, lástima e inseguridad a la discapacidad intelectual (Alfaro, 2017).

Como aspectos especialmente preocupantes, por su parte, surgen las referencias al estancamiento o bloqueo emocional que resultaría en una paralización vital en uno de los entrevistados y los deseos de desaparecer, aparentemente superados, relatados por otra entrevistada. Estos signos iniciales podrían transformarse en importantes problemáticas de salud mental si no se abordan preventivamente desde los especialistas y al interior del contexto familiar como una fuente informativa para sus integrantes y un aporte al fortalecimiento de la familia como fuente de apoyo emocional y guía en las pautas de crianza y contención.

En el caso de las dos hermanas extranjeras entrevistadas se pudieron visualizar problemáticas similares a las del grupo local, con mayor énfasis en el replegamiento endogámico como nicho protector y de resguardo frente a la sociedad dominante "debemos salir adelante y hacernos valer en este nuevo país", "cuidar que la miren doblemente diferente; como inmigrante y como especial (hace signo de entrecomillar con las manos)".

En síntesis, otorgándole voz y protagonismo a los/as hermanos/as de niños/as en situación de discapacidad, nos aproximamos a una comprensión del fenómeno de la DI como una problemática sistémica compleja, en tanto, no sólo se ven afectados estos/as niños/as y sus padres/madres, sino también todos/as aquellos/as que conforman el núcleo familiar del infante y que ven reorganizada y orientada su vida en torno a él. En el caso de los/as hermanos/as del estudio fue posible acceder a sus propias afectaciones y recorridos y, al mismo tiempo, localizar la problemática en una etapa vital, adolescencia en este caso, en una realidad social diversa y culturalmente situada (locales y extranjeros). Por tanto, se vuelve relevante hacer un abordaje que considere al sistema familiar en su totalidad reparando en sus respectivos subsistemas, no solo en términos de la intervención a realizar sino también en términos investigativos, puesto que todo lo que se produce y/o vivencia en dicho espacio de una u otra forma está directamente relacionado con el desarrollo de los (as) niños (as) en situación de discapacidad. Proyectar estudios con familia extendida será el siguiente paso en esta línea de investigación en AT y DI.

\section{Referencias}

Aguilar, D. H. y Chávez, R. R. (2013). La investigación biográfico-narrativa, una alternativa para el estudio de los docentes. Revista Electrónica Actualidades Investigativas en Educación, 13(3), 1-27. https://doi.org/10.15517/aie.v13i3.12026

Alfaro, J. E. (2017). El problema del enfoque médico de la discapacidad: Un desafío interdisciplinario entre salud, educación y el derecho. Revista Médica de Chile, 145, 678-681. https://doi.org/10.4067/So034-98872017000500018 
Álvarez, M. I. C., Alonso, M. Á. V. y Amor, A. M. (2016). La participación familiar es un requisito imprescindible para una escuela inclusiva. Revista Latinoamericana de Educación Inclusiva, 1O(1), 99-113. https://doi.org/10.4067/So718-73782016000100006

Andreucci, P. (noviembre, 2016). El protagonismo del contexto familiar en Atención Temprana: la experiencia de CET Chile. Ponencia realizada en las XXV Reunión Interdisciplinar sobre poblaciones con alto riesgo de deficiencias y IX Reunión Interdisciplinar sobre Discapacidades/Trastornos del Desarrollo y Atención Temprana. Pasado, Presente y Retos Pendientes de la Atención Temprana. Universidad Complutense de Madrid, Madrid.

Aparicio, R. y Tárraga, R. (2015). El ajuste conductual, emocional y social en hermanos de niños con un trastorno del espectro autista. Una revisión teórica. Papeles del Psicólogo, 36(3), 189197.

Arrabal, C. y Arizcun, J. (2005). Deficiencias, discapacidades del desarrollo y los servicios de neonatología. En F. Mulas y M. G. Millá (Eds.), Atención temprana: Desarrollo infantil, diagnóstico, trastornos e intervención (pp. 85-108). Madrid: Promolibro.

Bowlby, J. (1993). El vínculo afectivo. Madrid: Paidós Ibérica.

Cara-Díaz, M., Sola-Martínez, T., Aznar-Díaz, I. y Fernández-Martín, F. (2015). Análisis organizativo de las clases de inclusión escolar en Francia. Revista Latinoamericana de Ciencias Sociales, Niñez y Juventud, 13(2), 795-807.

https://doi.org/10.11600/1692715x.13217200314

Cecchini, S. y Martínez, R. (2011). Protección social inclusiva en América Latina: Una mirada integral, un enfoque de derechos. Santiago de Chile: CEPAL.

Escorsia-Mora, C., García-Sánchez, F., Sánchez-López, M., Orcajada, N. y Hernández-Pérez, E. (2018). Prácticas de atención temprana en el sureste de España: Perspectiva de profesionales y familias. Anales de Psicología, 34(3), 500-509.

https://doi.org/10.6018/analesps.34.3.311221

Federación Estatal de Asociaciones de Profesionales de Atención Temprana. (2000). Libro blanco de la atención temprana. Madrid: Centro Español de Documentación sobre Discapacidad.

Flores, P. J. y Porges, S. W. (2017). Group psychotherapy as a neural exercise: Bridging polyvagal theory and attachment theory. International Journal of Group Psychotherapy, 67(2), 202-222. https://doi.org/10.1080 / 00207284.2016.1263544

García-Sánchez, F. A., Escorcia-Mora, C. T., Sánchez-López, M. C., Orcajada, N. y HernándezPérez, E. (2014). Atención temprana centrada en la familia. Siglo Cero. Revista Española sobre Discapacidad Intelectual, 45(3), 6-27.

Giaconi, C., Pedrero, Z., y San Martín, P. (2017). La discapacidad: Percepciones de cuidadores de niños, niñas y jóvenes en situación de discapacidad. Psicoperspectivas, 16(1), 55-66. https://doi.org/10.5027/psicoperspectivas-Vol16-Issue 1-fulltext-822

González, R., Blanco, L. A. y Herrero, M. (2017). La percepción de padres y madres en el ejercicio del rol parental. International Journal of Developmental and Educational Psychology. Revista INFAD de Psicología, 6(1), 47-56. https://doi.org/10.17060/ijodaep.2014.n1.v6.716

González-Monteagudo, J. y Ochoa-Palomo, C. (2014). El giro narrativo en España: Investigación y formación con enfoques auto/biográficos. Revista Mexicana de Investigación Educativa, 19(62), 809-829.

Guralnick, M. J. (2011). Why early intervention works: A systems perspective. Infants and Young Children, 24(1), 6-28. https://doi.org/10.1097/IYC.obo13e3182002cfe

Guralnick, M. J. (2016). Early intervention: The integration of science and practice. Journal of Intellectual Disability Research, 60(7), 624-640. 
Guralnick, M. J. y Bennett, F. (1989). Eficacia de una intervención precoz en niños minusválidos y en situaciones de riesgo. En J. Haley (Ed.), Terapia no convencional (pp. 175-212). Madrid: Amorrortu.

Guralnick, M. J., Connor, R. T. y Johnson, L. C. (2011). The peer social networks of young children with Down syndrome in classroom programmes. Journal of Applied Research in Intellectual Disabilities, 24(4), 310-321. https://doi.org/10.1111/j.1468-3148.2010.00619.x

Hernández, M. I. (2015). El concepto de discapacidad: De la enfermedad al enfoque de derechos. Revista CES Derecho, 6(2), 46-59.

Iriarte, C. y Ibarrola-García, S. (2016). Revisión de estudios sobre la vivencia emocional de la discapacidad intelectual por parte de los hermanos. Estudios sobre Educación, 19, 53-75.

Kornblit, A. L. (2004). Historias y relatos de vida: Una herramienta clave en metodologías cualitativas. En A. L. Kornblit (Coord.), Metodologías cualitativas en ciencias sociales (pp. 1533). Buenos Aires: Editorial Bilblos.

Leal, L. (2008). Un enfoque de la discapacidad intelectual centrado en la familia. Madrid: FEAPS.

Lizasoain, O. y Onieva, C. E. (2010). Un estudio sobre la fratría ante la discapacidad intelectual. Psychosocial Intervention, 19(1), 89-99. https://doi.org/10.5093/in2010v19n 1a9

Lozano-Vicente, A. (2016). Los derechos del niño: Cuestiones sobre su fundamentación. Revista Latinoamericana de Ciencias Sociales, Niñez y Juventud, 14(1), 67-79.

Luciani-Conde, L. (2010). La protección social de la niñez: Subjetividad y posderechos en la segunda modernidad. Revista Latinoamericana de Ciencias Sociales, Niñez y Juventud, 8(2), 885899.

Martínez, B. (2011). Pobreza, discapacidad y derechos humanos: Aproximación a los costes extraordinarios de la discapacidad y su contribución a la pobreza desde un enfoque basado en los derechos humanos. Madrid: Ediciones Cinca.

McWilliam, R. (2016). Metanoia en atención temprana: Transformación a un enfoque centrado en la familia. Revista Latinoamericana de Inclusión Educativa, 10(1), 133-153. https://doi.org/10.4067/So7 18-73782016000100008

MINEDUC. (2007). Guía de apoyo técnico pedagógico: Necesidades educativas especiales asociadas al retraso del desarrollo y discapacidad intelectual. Santiago de Chile: MINEDUC.

Minuchin, S. y Nichols, M. P. y Lee, W. Y. (2007). Assessing families and couples: From symptom to system. Londres: Pearson Allyn and Bacon.

ONU. (2016). Comité sobre los derechos de las personas con discapacidad. Observaciones finales sobre el informe inicial de Chile. Recuperado de http://acnudh.org/wp-content/ uploads/2016/O4/CRPD_C_CHL_CO_1_23679_S.pdf

Palacios-Hernández, B. (2016). Alteraciones en el vínculo materno-infantil: Prevalencia, factores de riesgo, criterios diagnósticos y estrategias de evaluación. Revista de la Universidad Industrial de Santander, 48(2), 164-176. https://doi.org/10.18273/revsal.v48n2-2016001

Palacios Rizzo, A. y Bariffi, F. J. (2014). La discapacidad como una cuestión de derechos humanos. Una aproximación a la convención internacional sobre los derechos de las personas con discapacidad. Madrid: Ediciones Cinca.

Palomares-Ruiz, A., Sánchez-Navalón, B. y Garrote-Rojas, D. (2016). Educación inclusiva en contextos inéditos: La implementación de la pedagogía hospitalaria. Revista Latinoamericana de Ciencias Sociales, Niñez y Juventud, 14(2),1507-1522.

Peñas-Felizzola, O. (2013). Referentes conceptuales para la comprensión de la discapacidad. Revista Facultad de Medicina, 61(2), 205-212. 
Peregrina, E. B., Blanch, J. S., González, N. A. y Nicolau, J. R. (2015). Historias de vida y educación social: Una experiencia de investigación y formación. Tendencias Pedagógicas, 24, 71-84.

Pérez Lisboa, S. (2018). Práctica profesional en la sala de atención temprana. Un estudio descriptivo de su rol. Revista de Educación y Desarrollo, 48, 105-111.

Ponte, J. (2017). Buscando datos e indicadores para evaluar los servicios de atención temprana. Revista Española de Discapacidad, 5(1), 77-98. https://doi.org/10.5569/2340-5 104.05.01.05

Riessman, C. K. (2008). Narrative methods for the human sciences. Londres: Sage Publications.

Ruiz Aparicio, R. y Tárraga Mínguez, R. (2015). El ajuste conductual, emocional y social en hermanos de niños con un trastorno del espectro autista. una revisión teórica. Papeles del Psicólogo, 36(3),189-197.

Saavedra, C. (2015). ¿Qué es Chile crece contigo? Santiago de Chile: Unidad Chile Crece Contigo.

Sánchez, P. (2015). Primera infancia y discapacidad en Chile: Revisión y enfoque actual a los programas de gobierno. Revista Médica Clínica Las Condes, 26(4), 520-526. https://doi.org/10.1016/j.rmclc.2015.06.026

Sarasa, M. C. (2014). Experiencias de enseñanza y de aprendizaje mediante la investigación biográfica y la indagación narrativa. Revista de Educación, 7, 157-170.

Schriewer, K. y Agea, J. (2015). Cuestiones prácticas en cuanto a la elaboración de relatos biográficos. Quaderns-e de l'Institut Català d'Antropologia, 20(1), 114-131.

Simón, C., Giné, C. y Echeita, G. (2016). Escuela, familia y comunidad: Construyendo alianzas para promover la inclusión. Revista Latinoamericana de Inclusión Educativa, 10(1), 25-42. https://doi.org/10.4067/S07 18-73782016000100003

Turner, B. (2001). Disability and the sociology of the body. En G. Albrecht, K. Seelman y M. Bury (Eds.), Handbook of disability studies (pp. 252-267). Thousand Oaks, CA: SAGE.

Valverde, D. (2017). El estado actual de la investigación y la intervención sobre la vivencia emocional de los hermanos ante la discapacidad intelectual. Redes, 35, 71-82.

Veerman, E. R., Cuevas, P. G. y Arizcun-Pineda, J. (2005). La formación de los profesionales de la atención temprana. En P. Gútiez (Ed.), Atención temprana: Prevención, detección e intervención en el desarrollo (O-6 años) y sus alteraciones (pp. 935-953). Madrid: Editorial Complutense.

Vegas E., Cerdán-Infantes, P., Dunkelberg, E. y Molina, E. (2006). Evidencia internacional sobre políticas de la primera infancia que estimulen el desarrollo infantil y faciliten la inserción laboral femenina. Recuperado de: http://www.oei.es/inicial/ politicas_infanciadesarrollo.pdf

Viloria, C. A. y González, A. F. (2017). Las prácticas de crianza de los padres: Su influencia en las nuevas problemáticas en la primera infancia. Revista de Educación Inclusiva, 9(1), 30-42.

\section{Breve CV de las autoras}

\section{Paola Andreucci Annunziata}

Psicóloga y Licenciada en Psicología por la Universidad de Chile. Magíster en Ciencias de la Educación por la Pontificia Universidad Católica de Chile (PUC). Doctora en Ciencias de la Educación por la PUC. Académica e Investigadora de la PUC. Directora Centro de Investigación en Educación Universidad Gabriela Mistral (CIE-UGM). Directora Centro de Estimulación Temprana Universidad Gabriela Mistral (CET-UGM). con estudios de doctorado en Investigación en Psicoterapia (PUC, Universidad de Chile, Universidad de Heidelberg). Se interesa por la educación inclusiva, la problemática de la 
equidad desde la atención temprana hasta la educación superior y el aprendizaje socioemocional desde perspectivas narrativas, dialogales y dialógicas. ORCID ID:

https://orcid.org/O000-0002-4438-01 19. Email: paola.andreucci@ugm.cl

\section{Camila Morales Cabello}

Socióloga y Licenciada en Sociología por la Universidad Academia de Humanismo Cristiano. Master en Educación por la Universidad Nacional de La Plata. Asistente de Investigación del Centro de Investigación en Educación (CIE-UGM). ORCID ID: https://orcid.org/0000-0003-02 17-9721.Email: camila.morales@ugm.cl 